\title{
Front Matter: Volume 8851
}

, "Front Matter: Volume 8851," Proc. SPIE 8851, X-Ray Nanoimaging: Instruments and Methods, 885101 (11 October 2013); doi: $10.1117 / 12.2045770$

SPIE Event: SPIE Optical Engineering + Applications, 2013, San Diego, California, SPIE. United States 


\section{PROCEEDINGS OF SPIE}

\section{$X$-Ray Nanoimaging: Instruments and Methods}

Barry Lai

Editor

28-29 August 2013

San Diego, California, United States

Sponsored and Published by

SPIE 
The papers included in this volume were part of the technical conference cited on the cover and title page. Papers were selected and subject to review by the editors and conference program committee. Some conference presentations may not be available for publication. The papers published in these proceedings reflect the work and thoughts of the authors and are published herein as submitted. The publisher is not responsible for the validity of the information or for any outcomes resulting from reliance thereon.

Please use the following format to cite material from this book:

Author(s), "Title of Paper," in X-Ray Nanoimaging: Instruments and Methods, edited by Barry Lai, Proceedings of SPIE Vol. 8851 (SPIE, Bellingham, WA, 2013) Article CID Number.

ISSN: 0277-786X

ISBN: 9780819497017

Published by

SPIE

P.O. Box 10, Bellingham, Washington 98227-0010 USA

Telephone +1 3606763290 (Pacific Time) · Fax +1 3606471445

SPIE.org

Copyright @ 2013 , Society of Photo-Optical Instrumentation Engineers.

Copying of material in this book for internal or personal use, or for the internal or personal use of specific clients, beyond the fair use provisions granted by the U.S. Copyright Law is authorized by SPIE subject to payment of copying fees. The Transactional Reporting Service base fee for this volume is $\$ 18.00$ per article (or portion thereof), which should be paid directly to the Copyright Clearance Center (CCC), 222 Rosewood Drive, Danvers, MA 01923. Payment may also be made electronically through CCC Online at copyright.com. Other copying for republication, resale, advertising or promotion, or any form of systematic or multiple reproduction of any material in this book is prohibited except with permission in writing from the publisher. The CCC fee code is $0277-786 \mathrm{X} / 13 / \$ 18.00$.

Printed in the United States of America.

Publication of record for individual papers is online in the SPIE Digital Library.

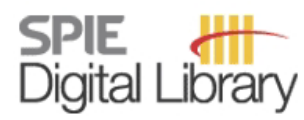

SPIEDigitalLibrary.org

Paper Numbering: Proceedings of SPIE follow an e-First publication model, with papers published first online and then in print and on CD-ROM. Papers are published as they are submitted and meet publication criteria. A unique, consistent, permanent citation identifier (CID) number is assigned to each article at the time of the first publication. Utilization of CIDs allows articles to be fully citable as soon as they are published online, and connects the same identifier to all online, print, and electronic versions of the publication. SPIE uses a six-digit CID article numbering system in which:

- The first four digits correspond to the SPIE volume number.

- The last two digits indicate publication order within the volume using a Base 36 numbering

system employing both numerals and letters. These two-number sets start with 00, 01, 02, 03, 04,

05, 06, 07, 08, 09, 0A, OB ... 0Z, followed by 10-1Z, 20-2Z, etc.

The CID Number appears on each page of the manuscript. The complete citation is used on the first page, and an abbreviated version on subsequent pages. Numbers in the index correspond to the last two digits of the six-digit CID Number. 


\title{
Contents
}

\author{
vii Conference Committee \\ ix Introduction
}

\section{SCANNING PROBES I}

885102 Sub-100-nm 3D-elemental mapping of frozen-hydrated cells using the bionanoprobe (Invited Paper) [8851-1]

S. Chen, Argonne National Lab. (United States); Y. Yuan, J. Deng, R. Mak, Q. Jin, T. Paunesku, Northwestern Univ. (United States); S. C. Gleber, D. Vine, Argonne National Lab. (United States); C. Flachenecker, B. Hornberger, Carl Zeiss X-ray Microscopy, Inc. (United States); D. Shu, B. Lai, J. Maser, L. Finney, C. Roehrig, Argonne National Lab. (United States); J. VonOsinski, M. Bolbat, K. Brister, Northwestern Synchrotron Research Ctr. (United States); C. Jacobsen, Argonne National Lab. (United States); G. Woloschak, Northwestern Univ. (United States); S. Vogt, Argonne National Lab. (United States)

885104 Status of the Nanoscopium scanning nanoprobe beamline of Synchrotron Soleil [8851-3] A. Somogyi, K. Medjoubi, C. M. Kewish, V. Leroux, M. Ribbens, G. Baranton, F. Polack, J. P. Samama, Synchrotron SOLEIL (France)

885106 A next-generation in-situ nanoprobe beamline for the Advanced Photon Source [8851-5] J. Maser, B. Lai, Argonne National Lab. (United States); T. Buonassisi, Massachusetts Institute of Technology (United States); Z. Cai, S. Chen, L. Finney, S.-C. Gleber, R. Harder, C. Jacobsen, W. Liu, Argonne National Lab. (United States); C. Murray, IBM Thomas J. Watson Research Ctr. (United States); C. Preissner, C. Roehrig, V. Rose, D. Shu, D. Vine, S. Vogt, Argonne National Lab. (United States)

\section{FULL-FIELD MICROSCOPES}

885107 Development of achromatic full-field x-ray microscopy with compact imaging mirror system (Invited Paper) [8851-6] S. Matsuyama, Y. Emi, H. Kino, Y. Sano, Osaka Univ. (Japan); Y. Kohmura, K. Tamasaku, M. Yabashi, T. Ishikawa, SPring-8/RIKEN (Japan); K. Yamauchi, Osaka Univ. (Japan)

885109 Development of large-field high-resolution hard x-ray imaging microscopy and microtomography with Fresnel zone plate objective [8851-8]

Y. Suzuki, A. Takeuchi, Y. Terada, K. Uesugi, Japan Synchrotron Radiation Research Institute (Japan); S. Tamura, National Institute of Advanced Industrial Science and Technology (Japan) 
8851 OA Data-processing strategies for nano-tomography with elemental specification (Invited Paper) [8851-9]

Y. Liu, SLAC National Accelerator Lab. (United States); K. H. Cats, Utrecht Univ. (Netherlands); J. Nelson Weker, J. C. Andrews, SLAC National Accelerator Lab. (United States); B. M. Weckhuysen, Utrecht Univ. (Netherlands); P. Pianetta, SLAC National Accelerator Lab. (United States)

$8851 \mathrm{OB}$ Identifying and managing radiation damage during in situ transmission $\mathrm{x}$-ray microscopy of Li-ion batteries [8851-10]

J. Nelson, SLAC National Accelerator Lab. (United States); Y. Yang, Stanford Univ. (United States); S. Misra, BASF Nederlands B.V. (Netherlands); J. C. Andrews, SLAC National Accelerator Lab. (United States); Y. Cui, Stanford Univ. (United States); M. F. Toney, SLAC National Accelerator Lab. (United States)

8851 OC Development of in-situ full-field spectroscopic imaging analysis and application on Li-ion battery using transmission $x$-ray microscopy [8851-11]

Y. K. Chen-Wiegart, J. Wang, J. Wang, Brookhaven National Lab. (United States)

8851 OD Development of in-line furnace for in-situ nanoscale resolution $\mathbf{x}$-ray microscopy [8851-12] C. Eng, Brookhaven National Lab. (United States) and Stony Brook Univ. (United States);

Y. K. Chen-Wiegart, J. Wang, Brookhaven National Lab. (United States)

\section{NANOFOCUSING OPTICS}

8851 OG Design optimization of ultra-precise elliptical mirrors for hard x-ray nanofocusing at Nanoscopium [8851-15]

C. M. Kewish, F. Polack, Synchrotron SOLEIL (France); R. Signorato, Bruker ASC GmbH (Germany); A. Somogyi, Synchrotron SOLEIL (France)

\section{SCANNING PROBES II}

8851 OK Development of an in-vacuum x-ray microscope with cryogenic sample cooling for beamline P11 at PETRA III [8851-19]

A. Meents, B. Reime, N. Stuebe, P. Fischer, M. Warmer, D. Goeries, J. Roever, J. Meyer, J. Fischer, A. Burkhardt, Deutsches Elektronen-Synchrotron (Germany); I. Vartiainen,

P. Karvinen, C. David, Paul Scherrer Institut (Switzerland)

8851 OL NanoMAX: a hard x-ray nanoprobe beamline at MAX IV [8851-20]

U. Johansson, Lund Univ. (Sweden); U. Vogt, KTH Royal Institute of Technology (Sweden);

A. Mikkelsen, Lund. Univ. (Sweden)

8851 OM Preliminary design of a zone plate based hard X-ray monochromatic diffraction nanoprobe for materials studies at APS [8851-21]

Z. Cai, W. Liu, J. Z. Tischler, D. Shu, R. Xu, O. Schmidt, Argonne National Lab. (United States) 
8851 OP Simultaneous fast scanning XRF, dark field, phase-, and absorption contrast tomography [8851-24]

K. Medjoubi, Synchrotron SOLEIL (France); A. Bonissent, Ctr. de Physique des Particules de Marseille (France) and CNRS/IN2P3 (France); N. Leclercq, F. Langlois, P. Mercère, A. Somogyi, Synchrotron SOLEIL (France)

$88510 Q$ The Maia detector array and $x$-ray fluorescence imaging system: locating rare precious metal phases in complex samples [8851-25]

C. G. Ryan, Commonwealth Scientific and Industrial Research Organisation (Australia); D. P. Siddons, Brookhaven National Lab. (United States); R. Kirkham, Commonwealth Scientific and Industrial Research Organisation (Australia); Z. Y. Li, Brookhaven National Lab. (United States); M. D. de Jonge, D. Paterson, Australian Synchrotron (Australia); J. S. Cleverley, Commonwealth Scientific and Industrial Research Organisation (Australia); A. Kuczewski, Brookhaven National Lab. (United States); P. A. Dunn, M. Jensen, Commonwealth Scientific and Industrial Research Organisation (Australia);

G. De Geronimo, Brookhaven National Lab. (United States); D. L. Howard, Australian Synchrotron (Australia); B. Godel, Commonwealth Scientific and Industrial Research Organisation (Australia); K. A. Dyl, Commonwealth Scientific and Industrial Research Organisation (Australia) and Curtin Univ. (Australia); L. A. Fisher, R. H. Hough, S. J. Barnes, Commonwealth Scientific and Industrial Research Organisation (Australia); P. A. Bland, Curtin Univ. (Australia); G. Moorhead, Commonwealth Scientific and Industrial Research Organisation (Australia); S. A. James, K. M. Spiers, Australian Synchrotron (Australia); G. Falkenberg, U. Boesenberg, G. Wellenreuther, Hasylab, Deutsches ElektronenSynchrotron (Germany)

\section{NOVEL NANOIMAGING METHODS}

8851 OT A universal measure for coherence requirements in diffractive imaging [8851-28]

B. Chen, B. Abbey, E. Balaur, G. van Reissen, M. Junker, M. W. M. Jones, La Trobe Univ. (Australia); A. G. Peele, La Trobe Univ. (Australia) and Australian Synchrotron (Australia); C. T. Putkunz, The Univ. of Melbourne (Australia); D. Vine, Advanced Photon Source, Argonne National Lab. (United States); H. M. Quiney, The Univ. of Melbourne (Australia); K. A. Nugent, La Trobe Univ. (Australia)

\section{LAB-BASED INSTRUMENTS}

8851 oW Optimized cavity-enhanced x-ray sources for x-ray microscopy (Invited Paper) [8851-31] J. M. J. Madey, E. B. Szarmes, M. R. Hadmack, Univ. of Hawai'i at Manoa (United States); B. T. Jacobson, RadiaBeam Technologies, LLC (United States); J. M. D. Kowalczyk, P. Niknejadi, Univ. of Hawaili at Manoa (United States)

8851 OY Tabletop coherent diffractive imaging of extended objects in transmission and reflection geometry [8851-33] M. D. Seaberg, B. Zhang, D. E. Adams, D. F. Gardner, H. C. Kapteyn, M. M. Murnane, Univ. of Colorado at Boulder (United States) 
$88510 Z$ High efficiency $x$-ray nanofocusing by the blazed stacking of binary zone plates [8851-34] I. Mohacsi, P. Karvinen, I. Vartiainen, A. Diaz, Paul Scherrer Institut (Switzerland); A. Somogyi, C. M. Kewish, P. Mercere, Synchrotron SOLElL (France); C. David, Paul Scherrer Institut (Switzerland)

885112 Analysis of impact of sintering temperature on microstructure of LSCF-SDC composite cathodes using nano-CT [8851-37]

Y. Guan, X. Pan, G. Liu, Z. Liang, S. Chen, X. Zhang, Y. Xiong, Y. Tian, C. Xia, Univ. of Science and Technology of China (China)

885113 Reconstruction of limited-angle and few-view nano-CT image via total variation iterative reconstruction [8851-38]

Z. Liang, Y. Guan, G. Liu, R. Bian, X. Zhang, Y. Xiong, Y. Tian, Univ. of Science and Technology of China (China)

885117 A soft $x$-ray beamline for quantitative nanotomography using ptychography [8851-43] G. A. van Riessen, M. Junker, N. W. Phillips, Australian Research Council Ctr. of Excellence in Coherent X-Ray Science (Australia) and La Trobe Univ. (Australia); A. G. Peele, Australian Research Council Ctr. of Excellence in Coherent X-Ray Science (Australia), La Trobe Univ. (Australia), and Australian Synchrotron (Australia)

885119 Recent advances in use of atomic layer deposition and focused ion beams for fabrication of Fresnel zone plates for hard $\mathbf{x}$-rays [8851-45]

K. Keskinbora, Max-Planck Institut für Intelligente Systeme (Germany); A.-L. Robisch, Univ. Göttingen (Germany); M. Mayer, C. Grévent, Max-Planck Institut für Intelligente Systeme (Germany); A. V. Szeghalmi, Friedrich-Schiller-Univ. Jena (Germany); M. Knez, CIC nanoGUNE Consolider (Spain) and Ikerbasque, Basque Foundation for Science (Spain); M. Weigand, Max-Planck Institut für Intelligente Systeme (Germany); I. Snigireva, A. Snigirev, European Synchrotron Radiation Facility (France); T. Salditt, Univ. Göttingen (Germany); G. Schütz, Max-Planck Institut für Intelligente Systeme (Germany)

8851 1D Full-field $x$-ray nano-imaging at SSRF [8851-49]

B. Deng, Y. Ren, Y. Wang, G. Du, H. Xie, T. Xiao, Shanghai Institute of Applied Physics (China)

Author Index 


\section{Conference Committee}

Program Track Chair

Carolyn A. MacDonald, University at Albany (United States)

Conference Chair

Barry Lai, Argonne National Laboratory (United States)

Conference Program Committee

Michael Feser, Carl Zeiss X-ray Microscopy (United States)

Hans M. Hertz, Royal Institute of Technology (Sweden)

Ian McNulty, Argonne National Laboratory (United States)

David Paterson, Australian Synchrotron (Australia)

Christian G. Schroer, Technische Universität Dresden (Germany)

Andrea Somogyi, Synchrotron SOLEIL (France)

Kazuto Yamauchi, Osaka University (Japan)

Session Chairs

1 Scanning Probes I

Barry Lai, Argonne National Laboratory (United States)

2 Full-Field Microscopes

David J. Paterson, Australian Synchrotron (Australia)

3 In-situ TXM Studies

Benjamin Hornberger, Carl Zeiss X-ray Microscopy (United States)

4 Nanofocusing Optics

Christian G. Schroer, Technische Universität Dresden (Germany)

5 Beam Characterization

Christian G. Schroer, Technische Universität Dresden (Germany)

6 Scanning Probes II

Andrea Somogyi, Synchrotron SOLEIL (France)

7 Fast Instrumentation

Stefan Vogt, Argonne National Laboratory (United States) 
8 Novel Nanoimaging Methods

Ian McNulty, Argonne National Laboratory (United States)

9 Lab-based Instruments

Hans M. Hertz, Royal Institute of Technology (Sweden) 


\section{Introduction}

It has been a pleasure to organize the SPIE X-ray Nanoimaging Conference. It started from a simple office visit by a longtime colleague, Ali Khounsary, who had organized numerous SPIE x-ray conferences. With Ali's encouragement and advice, I started to contact potential members of the program committee. Surprisingly everyone accepted and agreed to serve, and an international program committee was quickly established. However the most remarkable occurrence had to be the enthusiastic response from the x-ray nanoimaging community: 48 original and exciting abstracts were submitted within a short time. It demonstrated the breadth and depth of the x-ray nanoimaging community in general, which had seen substantial growth recently due to construction of new and upgrade of existing synchrotron facilities worldwide.

The conference was organized into two days of oral presentations, with a lively evening poster session in between. Topics included scanning probes, full-field microscopes, in-situ studies, nanofocusing optics, beam characterization, fast instrumentation, novel methods, and lab-based instruments. Overall the presentations were exciting, the audiences were engaging, and it was particularly gratifying to see new researchers participating at the conference. Special thanks to the program committee members for their volunteer but hard work, the SPIE staff for their professional support, session chairs for managing the thoughtful discussions, authors and presenters for sharing the latest developments, and all the active participants, who together made this a very rewarding experience.

Barry Lai 